\title{
Prácticas de mejora para el proceso de acompañamiento pedagógico
}

Improvement practices for the pedagogical accompaniment process

\author{
Volumen 21, Número 2 \\ Mayo - Agosto \\ pp. 1-24
}

\section{Daniel San Martín Cantero \\ Reina San Marín Aedo \\ Sergio Pérez Morales Jessica Bórquez Mella}

\section{Citar este documento según modelo APA}

San Martín Cantero, Daniel., San Martín Aedo, Reina., Pérez Morales, Sergio., y Bórquez Mella, Jessica. (2021). Prácticas de mejora para el proceso de acompañamiento pedagógico. Revista Actualidades Investigativas en Educación, 21(2), 1-24. Doi. 10.15517/aie.v21i2.46783 


\title{
Prácticas de mejora para el proceso de acompañamiento pedagógico
}

\author{
Improvement practices for the pedagogical accompaniment process
}

\author{
Daniel San Martín Cantero ${ }^{1}$ \\ Reina San Martín Aedo ${ }^{2}$ \\ Sergio Pérez Morales ${ }^{3}$ \\ Jessica Bórquez Mella ${ }^{4}$
}

\begin{abstract}
Resumen: Este artículo se deriva de un proyecto de investigación sobre experiencias de acompañamiento vivenciadas por estudiantes de pedagogía en su práctica profesional final en la región de La Araucanía, Chile. Fue desarrollado durante el periodo 2018-2019 por investigadores de la Universidad Católica de Temuco. El propósito fue describir problemáticas y criterios de mejoramiento durante el acompañamiento en la formación inicial docente. Los antecedentes sitúan el acompañamiento como experiencia de aprendizaje que puede resultar problemática para estudiantes que no cuentan con una orientación pedagógica oportuna. Se utilizó el método cualitativo y se entrevistó a 15 estudiantes de internado pedagógico. Las entrevistas fueron codificadas de acuerdo al enfoque de Teoría Fundamentada. Los resultados fueron categorías asociadas con gestión de la universidad, relación profesorado tutor-estudiantes y características socioculturales como problemáticas en el acompañamiento. Además, se exponen criterios para mejorar el acompañamiento pedagógico relacionados con selección de tutores y tutoras e incentivos para reconocer su labor. A modo de conclusión, se advierte un estatus artesanal del acompañamiento, que genera desamparo en estudiantes. De este modo, se develan exigencias formativas opuestas a las necesidades de centros de práctica pedagógica. Se visibiliza la importancia de articular universidades y centros de prácticas para re-pensar los roles de las personas tutoras y el estudiantado. En consecuencia, se recomienda diseñar un perfil de tutor y tutoras que represente los intereses de la comunidad educativa para disminuir la brecha entre teoría y práctica.
\end{abstract}

Palabras clave: formación de docentes, tutoría, práctica pedagógica, supervisión docente.

Abstract: This article is derived from a research project on accompaniment experiences experienced by pedagogy students in their final professional practice in the region of La Araucania, Chile. It was developed during the 20182019 period by researchers from the Catholic University of Temuco. The purpose was to describe problems and criteria for improvement during the accompaniment in the initial teacher training. The antecedents place the accompaniment as a learning experience that can be problematic for students who do not have a timely pedagogical orientation. The qualitative method was used and 15 pedagogical boarding students were interviewed. The interviews were coded according to the Grounded Theory approach. The results were categories associated with university management, tutor-student relationship and sociocultural characteristics as problems in the accompaniment. In addition, criteria are set out to improve pedagogical support related to the selection of tutors and mentors and incentives to recognize their work. By way of conclusion, there is an artisanal status of the accompaniment, which generates helplessness in students. In this way, training demands are revealed that are contrary to the needs of pedagogical practice centers. The importance of articulating universities and practice centers to rethink the roles of tutors and students is made visible. Consequently, it is recommended to design a tutor profile that represents the interests of the educational community to reduce the gap between theory and practice.

Key words: teacher education, mentoring, student teaching, teacher supervision

\footnotetext{
${ }^{1}$ Universidad Católica de Temuco, Araucanía, Chile. Director Centro de Investigación Escolar y Desarrollo. Dirección electrónica: dsanmartin@uct.cl Orcid: http://orcid.org/0000-0002-6316-4411

2 Universidad Católica de Temuco, Araucanía, Chile. Investigador adjunto Centro de Investigación Escolar y Desarrollo. Dirección electrónica: reinasanmartin77@gmail.com Orcid: http://orcid.org/0000-0002-0238-5325

${ }^{3}$ Universidad Católica de Temuco, Araucanía, Chile. Académico Facultad de Educación. Dirección electrónica: smorales@uct.cl Orcid: http://orcid.org/0000-0003-4704-1507

4 Universidad Católica de Temuco, Araucanía, Chile. Académica Facultad de Educación. Dirección electrónica: iborquez@uct.cl Orcid: http://orcid.org/0000-0001-7954-5983
}

Artículo recibido: 19 de noviembre, 2020

Enviado a corrección: 19 de febrero, 2021

Aprobado: 22 de marzo, 2021 


\section{Introducción}

En Chile, la formación inicial docente se remonta a 1842 con la Escuela Normal de Preceptores (Núñez, 2002). En este período, la política educativa centra su interés sobre la pedagogía alemana (Mansilla, 2018). Este enfoque pedagógico ofrecía mejorar el aprendizaje de métodos de enseñanza para la formación inicial docente.

Hacia la primera década del siglo XX la formación docente se expande a todo el país mediante la instalación de escuelas normales. En esta época comienza una discusión en torno a los fundamentos filosóficos de la pedagogía. Según Cox y Gysling (1990), estas discusiones contribuirían a la adopción del pragmatismo americano representado en una pedagogía de base científica (Dewey, 1988). En consecuencia, las escuelas normales, en tanto instituciones formadoras, adquirirían un reconocido prestigio por la calidad de la formación docente. No obstante, esta trayectoria es interrumpida por la dictadura cívico-militar (1973-1990) mediante los decretos ley 179 de 1973 y el 353 de 1974, los que mandatan la paralización y posterior cierre de las Escuelas Normales respectivamente (Gimeno, 2014), y en los que se delega, en las universidades, la formación docente.

En relación con aspectos pedagógicos se considera que el saber profesional docente es esencialmente práctico (Alliaud, 2017). Esto se expresa en una racionalidad orientada por procedimientos pedagógicos rutinarios (Perrenoud, 2004). Por ejemplo, el diseño de la enseñanza da lugar a actividades y contenidos que resultan en aprendizajes prácticos (EirínNemiña, 2018). En efecto, los saberes pedagógicos responden a contextos de vida reales (Herrera y Martínez 2018; Páez, 2015). Esto supone que el conocimiento práctico del profesorado es producto de la experiencia pedagógica situada (Hevia y Fueyo, 2018; Méndez, 2012). Ciertamente, se trata de conocimiento amarrado al contexto y a la persona, además incluye normas, valores y creencias de la comunidad (Ávalos, 2004; Elías, 2015).

Todas estas cualidades forman saberes ineludibles para aquellos docentes que se inician en la pedagogía. Por esta razón, la práctica pedagógica o practicum es conditio sine qua non para el aprendizaje de saberes prácticos-pedagógicos (Bullough, 2000; Sánchez-Amaya y González-Melo, 2016).

En el ámbito formativo, la inmersión en contextos pedagógicos reales permite al estudiantado completar su formación inicial docente (Sánchez, 2016). Más bien es, una inmersión en la cultura escolar, oportunidad para probar aquellos saberes aprendidos en el espacio académico formativo. En este sentido, Tejada (2006) sostiene que la práctica profesional es un espacio de integración y encuentro entre teoría y práctica. 
Pese a que la práctica pedagógica aporta a la formación pedagógica, también contiene múltiples dificultades. Por ejemplo, Sánchez (2016) se refiere a la imposibilidad de seleccionar aquellos centros que tienen prácticas innovadoras. Por esta razón, es necesario mejorar la colaboración entre centros de práctica y estructuras organizacionales de la formación docente. Esto requiere de un sistema de apoyo para docentes 'neófitos' (Ávalos, 2004). Para ilustrar, articular procesos de evaluación entre instituciones formadoras y centros de práctica profesional. También se puede fortalecer la eficacia de las tutorías mediante entrenamiento a las personas tutoras (Slavin, 1991). Del mismo modo Adaros (2014) concluye que hay una debilidad en los juicios emitidos por las personas tutoras, que da cuenta de escasa fundamentación en las ciencias de la educación y en los marcos curriculares.

Parte de las dificultades del profesorado novel se refieren a sentimientos de agrado o desagrado, de seguridad o inseguridad personal, esto provoca que el desarrollo profesional se caracterice por generar un recorrido irregular e incierto (Marcelo, 2008). A esto se suma la escasa supervisión de prácticas de enseñanza (Carnoy, 2010), considerando que en Chile futuros profesores y profesoras tienen muy poca formación para enseñar el programa nacional: “...sé un montón de cosas sobre teoría de enseñanza y sobre el español, pero casi nada acerca de cómo enseñar español" (Carnoy, 2010, p. 140). Esto se traduce en temores y dudas; percepción de incompetencia científica, investigadora y didáctica; inseguridad; necesidad de ayuda; inexperiencia; falta de consejo, profesionalidad y compañerismo; entre otros.

En este contexto, surge la siguiente pregunta: ¿cuáles son las problemáticas en el acompañamiento pedagógico experimentado por estudiantes durante sus prácticas profesionales? Por tanto, se plantea el objetivo de describir dificultades y criterios de mejoramiento durante el proceso de acompañamiento pedagógico. Esto permitirá comprender si el tipo de acompañamiento que el estudiantado de pedagogía recibe se ajusta a criterios de calidad que le permita tener una inserción docente exitosa.

\section{Referente Teórico}

En términos relacionales, el acompañamiento demanda un vínculo entre quien aprende y quien enseña, el cual da paso a complejas estructuras de relación. Desde este punto de vista, hay maestros y maestras que han destruido psicológicamente a sus discípulos y discípulas. También ha habido aprendices que han traicionado a sus maestros o maestras (Steiner, 2011). Esta fuente relacional también da paso a un aprendizaje mutuo, es decir no solo un discípulo o discípula aprende, sino también su maestro o maestra mientras enseña. 
Por otra parte, en el aprendizaje se deja ver una relación de imitación. A propósito de la auténtica enseñanza representada por la imitatio de un acto trascendente. Es decir, la imitación supone para cada estudiante un acto de descubrimiento y aprendizaje, en donde la autoridad pedagógica está en servir de modelo.

Así, la relación profesor o profesora-estudiante se traduce en una relación de autoridad, confianza, empatía, traición, conflicto, demostración, entre otras tramas relacionales que se articulan en un trayecto de acompañamiento. Acompañamiento que implica la tutela de la persona docente sobre su estudiante. Benoit (2021) concluye que la empatía y la solidaridad son habilidades sociales imprescindibles para construir confianza entre personas tutoras.

En efecto, la tutela cultiva el cuidado, entonces las personas docentes se convierten en tutores o tutoras que protegen y dirigen a sus estudiantes (Ariza y Ocampo, 2005). Por consiguiente, la tutoría debe entenderse como responsabilidad del profesorado en un escenario de interacciones personalizadas entre docente y estudiante (García Nieto, 2008).

\subsection{Tutorías y modelos de acompañamiento}

Durante el siglo XIX el tutor o tutora debía enseñar a sus estudiantes cómo usar sus mentes. A enseñar cómo pensar, no enseñar qué pensar (Moore, 1968). Este es un principio que ha orientado los modelos de acompañamiento en occidente. Sin embargo, teóricamente se plantean diversos formatos de tutoría que convergen en mejorar la calidad de la formación. Por ejemplo, la tutoría individual o asesoría personal (Arguis et al., 2011). En esta un profesortutor o profesora-tutora pretende conocer la situación personal de cada estudiante, para así ayudar y orientar la planificación y ejecución de sus tareas escolares. Otra tutoría es la realizada en un grupo de estudiantes. En esta modalidad su profesor o profesora-tutor o tutora apoya la orientación del currículo y la participación en el centro educativo (Ariza y Ocampo, 2005). Además de la tutoría en grupo se incluyen las tutorías técnicas, de la diversidad, de prácticas en empresas, entre otras descritas en la Tabla 1. 
Tabla 1

Tipificación de tutorías y principales rasgos

\begin{tabular}{ll}
\hline \multicolumn{1}{c}{ Tipo de tutoría } & \multicolumn{1}{c}{ Rasgos } \\
\hline Individual & $\begin{array}{l}\text { Trabaja sobre la autoestima del estudiantado, estimula la toma de } \\
\text { responsabilidades, desafíos y expresión de emociones. } \\
\text { Informa sobre condiciones y características del centro educativo y de } \\
\text { alumnos y alumnas. }\end{array}$ \\
Técnica o académica & $\begin{array}{l}\text { Es desempeñada por profesorado no designado como personas tutoras, } \\
\text { pero que se orienta a su seguimiento. }\end{array}$ \\
De la diversidad & Considera capacidades y ritmos de aprendizajes. \\
De práctica en empresas & $\begin{array}{l}\text { Se realiza por docentes cuando supervisan prácticas pedagógicas } \\
\text { profesionales }\end{array}$ \\
Profesional & Se expande hacia institutos, colegios, mundo empresarial y laboral \\
Estratégica & Enseña estrategias de aprendizaje al estudiante. Su objetivo es el éxito \\
& del aprendizaje
\end{tabular}

Fuente: Adaptado de Arbizu, Lobato y Castillo (2005); Cano (2008); Hock y Pulvers (2001).

Las tutorías muestran beneficios tanto para estudiantes como para tutores y tutoras (Cid, Pérez y Sarmiento, 2011). Otorgan beneficios a personas tutoras desde el desarrollo profesional y personal expresado en: aprendizaje y reflexión sobre la propia práctica, conocimiento de nuevas didácticas y mejoramiento de habilidades comunicativas y satisfacción personal. Por cuanto, cada estudiante se beneficia del apoyo al desarrollo profesional, colaboración en la construcción de aprendizajes y la socialización en la cultura escolar.

Si bien es cierto que la tutoría implica acompañamiento, este último puede formar parte de un plan general articulado por objetivos institucionales e incluso políticas educativas. En un sentido amplio, el acompañamiento a prácticas pedagógicas refiere a la acción realizada por todos aquellos que acompañan a docentes en formación, al inicio o durante el desarrollo del ejercicio de la enseñanza (Lunenberg, Dengerink y Korthagen, 2014).

El acompañamiento es una oportunidad para facilitar el crecimiento profesional del profesorado novel. Se trata de oportunidades de observar y comprometerse con buenas prácticas educativas, llevarlas a cabo, recibir ayuda y asesoramiento en forma de feedback con nuevas ideas y posibilidades de mejoramiento (Marcelo, 2008). A pesar que en situaciones de acompañamiento la relación entre la persona tutora y su estudiante se ve como un vínculo jerárquico, en donde las experiencias de quien ofrece la tutoría son trasvasijadas a docentes principiantes, sin otorgar instancias de diálogo para un crecimiento profesional reflexivo.

A raíz de lo anterior, se sugiere detenerse en la preparación de tutores y tutoras eficaces como personas modelos y consejeras (Munchi, 2018). Esto desafía las normas establecidas de la enseñanza, requiere de importantes recursos humanos y materiales para implementar 
esta innovación. Quienes desarrollan las tutorías requieren formación específica y seguimiento de su nuevo rol. Junto a esto, hace falta investigar sobre el desempeño, competencias del profesorado tutor en el acompañamiento pedagógico.

Una persona docente tutora debe desarrollar características particulares, que la habiliten para ejecutar su labor. De acuerdo con Harvard Business School (2004), las competencias que debe poseer una persona docente tutora para un acompañamiento exitoso se enmarca en el desarrollo de tres dimensiones, como se explica en la Tabla 2.

Tabla 2

Competencias de un profesor o profesora-tutor o tutora para un acompañamiento exitoso Dimensiones Características

Logros

Conocimiento del estudiante

Recursos relacionales

Fuente: Harvard Business School (2004)

En cuanto a los modelos o programas de acompañamiento/inducción, Vonk (1994) presenta una clasificación en donde distingue cuatro modelos de apoyo proporcionados a docentes debutantes para su desarrollo profesional:

a. Modelo nada o húndete (swim or sink): se trata del más frecuente en los establecimientos escolares, se anula la posibilidad de interacción con los pares. Se asume que el desarrollo profesional es un asunto de responsabilidad individual. En este modelo, la aceptación del grupo dependerá de que un nuevo docente demuestre su capacidad.

b. Modelo de compañerismo (collegial): supone cierta relación del profesor o profesora con alguno de los pares, pero de modo inestructurado e informal. En este contexto, un «colega» docente, a petición del interesado, puede proporcionarle ayuda para algunos asuntos específicos del funcionamiento docente (manejo del aula, técnicas docentes).

c. Modelo de adquisición de competencias necesarias (mandatory competency): se basa en el supuesto de que existe una serie de destrezas universales que caracterizan la docencia eficiente. Se establece una relación formal y jerárquica entre un docente novato o novata y una experta o experto. Este último orienta al primero hacia la adquisición de competencias básicas (dominio del aula, técnicas de enseñanza y materias a enseñar).

d. Modelo formalizado de mentor-protegido (formalized mentor-protegee): supone la existencia de una persona mentora entrenada, capaz de ayudar a docentes debutantes a estructurar y guiar sus procesos de aprendizaje profesional. Les convierte 
progresivamente en profesionales autónomos y auto-dirigidos; el foco de la acción de apoyo está en el desarrollo profesional del debutante. Para esto se desarrollan tres dimensiones básicas: personal, saberes y destrezas, y ecológico-contextuales.

Además de los modelos de Vonk (1994) también está el acompañamiento reflexivo (Donnay y Charlier, 2006). Este acompañamiento sugiere que la adquisición de aprendizajes para la formación del profesorado surge desde situaciones y problemas en el contexto real de la enseñanza. Por lo tanto, sobre este escenario problemático se construyen procesos de reflexión sobre la enseñanza.

\section{Metodología}

En este estudio cualitativo se difunden resultados de una investigación llevada a cabo durante el periodo 2018-2019.

\subsection{Enfoque}

Para indagar en las experiencias de acompañamiento, y desde la visión de sus protagonistas, se utilizó el método cualitativo (Canales, 2017; Denzin y Lincoln 2015; Flick, 2019). El diseño empleado fue Teoría Fundamentada, el que facilita la identificación de categorías emergentes (Denzin y Lincoln, 2015; Strauss y Corbin, 2002). Se trata de un diseño que permite una teorización sustantiva del material empírico para así ofrecer explicaciones situadas sobre el fenómeno en estudio (Flick, 2019). Su uso se justifica en el desarrollo e interpretación de relaciones conceptuales en torno a las categorías emergentes: problemáticas del acompañamiento y criterios de mejoramiento desde la experiencia de estudiantes en práctica profesional docente.

\subsection{Unidades de análisis}

Quienes participaron de esta investigación fueron estudiantes en práctica profesional docente de la Universidad Católica de Temuco, Chile. Como criterio de selección de participantes se releva la experiencia en prácticas pedagógicas. En particular, se seleccionó a 15 estudiantes de internado pedagógico ${ }^{6}$ (en adelante estudiantes IP) de todas las carreras de

\footnotetext{
${ }^{6}$ El internado pedagógico corresponde a la práctica pedagógica final en escuelas o liceos que forman parte de los centros de prácticas asignados al estudiantado. Durante este periodo, los estudiantes tienen dedicación exclusiva a la práctica pedagógica.
} 
la Facultad de Educación. Así, el muestreo fue intencional (Ruiz, 2012), basado en los criterios de la Tabla 3.

Tabla 3

Características del estudiantado participante, Universidad Católica de Temuco-Chile, 2020

\begin{tabular}{ll}
\hline Criterios & Rasgos \\
\hline Participantes & Estudiantes en práctica profesional docente \\
\hline Género & $\begin{array}{l}\text { Mujeres 12 } \\
\text { Hombres 3 }\end{array}$ \\
\hline \multirow{2}{*}{ Criterios de inclusión } & $\begin{array}{l}\text { Estudiantes de pedagogía en etapa de internado pedagógico } \\
\text { Prácticas pedagógicas en instituciones escolares }\end{array}$ \\
\hline \multirow{2}{*}{ Criterios de exclusión } & Estudiantes de pedagogía en etapa inicial \\
\hline Total participantes & Prácticas pedagógicas en instituciones no escolares \\
\hline
\end{tabular}

Fuente: Elaboración propia (2020)

Para el resguardo de la confidencialidad de la información e identidad de las personas participantes (Estay, 2011), se entregó un consentimiento informado que expresó el compromiso del investigador, procedimientos de la investigación, y voluntad de participación.

\subsection{Técnicas de recolección}

Se utilizó una entrevista temática (Valles, 1997), que se aplicó a cada participante. Este tipo de técnica permite explorar áreas donde las personas entrevistadas perciben contradicciones y dificultades (Banister, Burman, Parker, Taylor y Tindall, 2004), y así comprender formas particulares del fenómeno (acompañamiento) en su propia singularidad (Canales, 2013). Los temas que orientaron la conversación fueron: problemáticas experimentadas durante el proceso de acompañamiento, y sugerencias para mejorar el acompañamiento. Cada entrevista temática tuvo una duración aproximada de 60 minutos y se registró mediante una grabación de audio en formato digital. Luego, se incorporó a un software para análisis cualitativo de datos. Este software permitió codificar directamente los audios de las entrevistas temáticas, por lo cual no fue necesario transcribir toda la entrevista en audio.

\subsection{Procesamiento de Análisis}

El análisis de datos deviene de una tradición interpretacional (Trinidad, Carrero y Soriano, 2006) el que posibilita la compresión sobre la experiencia de acompañamiento como objeto particular dentro de la dimensión socioeducativa. Desde esta lógica analítica se compararon incidentes, propiedades y significados para levantar códigos interpretativos 
mediante un proceso de codificación abierta (San Martín, 2014; Strauss y Corbin, 2002; Maxwell, 2019). El resultado son categorías y subcategorías que conceptualizan las problemáticas experimentadas por estudiantes de IP.

Para la segmentación y articulación del material empírico se utilizó el Software Atlas-ti 7.5 (Gibbs, 2009; Woolf y Silver, 2018), ya que permite identificar relaciones conceptuales y elaborar modelos teóricos y categoriales, lo cual permite mejorar la calidad del análisis para la investigación educativa (San Martín, 2014).

\section{Resultados}

Los resultados de esta investigación correspondieron a las categorías problemáticas del acompañamiento y criterios de mejoramiento. A partir de estas categorías, se desarrolló el cuerpo narrativo expresado por estudiantes de IP e interpretado temáticamente mediante subcategorías y códigos que articularon la experiencia de acompañamiento. En la Tabla 4 se detallan las categorías Problemáticas del acompañamiento y Criterios de mejoramiento, las subcategorías relacionadas a cada categoría principal y códigos que permitieron el análisis de los datos.

Tabla 4

Categorías, subcategorías y códigos sobre experiencia de acompañamiento del estudiantado IP Universidad Católica de Temuco-Chile, 2020

\begin{tabular}{|c|c|c|c|}
\hline Categorías & & Subcategorías y códigos & \\
\hline \multirow{3}{*}{$\begin{array}{l}\text { Problemáticas } \\
\text { acompañamiento }\end{array}$} & \multirow{3}{*}{ del } & Gestión de la universidad & Falta de coordinación \\
\hline & & $\begin{array}{l}\text { Relación tutor o tutora-estudiante de } \\
\text { IE }\end{array}$ & $\begin{array}{l}\text { Falta de apoyo y ausencia de } \\
\text { tutoras y tutores en sala de } \\
\text { clases }\end{array}$ \\
\hline & & $\begin{array}{l}\text { Características socioculturales del } \\
\text { alumnado }\end{array}$ & $\begin{array}{l}\text { Desmotivación } \\
\text { aprendizaje }\end{array}$ \\
\hline $\begin{array}{l}\text { Criterios } \\
\text { mejoramiento }\end{array}$ & de & Selección de tutores y tutoras & $\begin{array}{l}\text { Perfil del profesor o profesora } \\
\text { Disposición para el } \\
\text { acompañamiento } \\
\text { Reconocimiento financiero y/o } \\
\text { profesional } \\
\text { Evaluación del desempeño del } \\
\text { profesor o profesora }\end{array}$ \\
\hline
\end{tabular}

Fuente: Elaboración propia (2020) 


\subsection{Problemáticas del acompañamiento}

En esta categoría se muestran las dificultades experimentadas por estudiantes de IP durante el acompañamiento recibido en sus prácticas pedagógicas. Estas dificultades se vinculan con la gestión de la universidad, relación entre el profesorado, tutores o tutoras y características socioculturales del alumnado de establecimientos escolares.

\subsubsection{Gestión de la universidad}

En esta Subcategoría se evidencia el modo en que la gestión de la universidad representa una dificultad para el acompañamiento que reciben estudiantes de IP. De este modo, se muestra escasa coordinación con escuelas, lo cual se expresa en la desvinculación entre personas supervisoras, personas tutoras y estudiantes de IP.

En atención a la escasa coordinación entre supervisión y tutoría es posible señalar que las exigencias de ambos se muestran incongruentes para el estudiantado de IP. Por esto, el estudiante de IP debe responder de manera simultánea frente a demandas contrapuestas. La consecuencia de esta doble exigencia se debe a una desvinculación entre universidad y centro de práctica:

...yo tenía que preparar material para tres cursos, esos tres cursos [...], muchas horas de clases y material a la semana, y además de eso tenía que estar respondiendo con todas las exigencias de la universidad. Por ejemplo, tengo un diagnóstico..., es tu tiempo de diagnóstico y tengo un mes para levantar un diagnóstico, pero yo a la segunda semana tenía que estar haciendo clases. Entonces había una desvinculación entre lo que me pedía la universidad, y lo que me pedía el centro de práctica, lo que me pedía el profesor supervisor, y lo que me pedía el profesor tutor..., que me llevaba casi al desdoblamiento. (Entrevista 01:25).

De acuerdo con Gervais y Desrosiers (2005), se hace imprescindible fomentar saberes prácticos que permitan una cultura de la colaboración universidad-centros educativos. Estos momentos de intercambios colaborativos entre la tríada de protagonistas (supervisor y supervisora-profesorado tutor-estudiante en práctica); facilita la autonomía y control de la acción pedagógica en los estudiantes en práctica (Cornejo, 2014). Esto supone acortar las brechas entre la teoría y la práctica que ha caracterizado la formación profesional. Lo anterior concuerda con el estudio de González, Uvalle y Hernández (2017), quienes concluyen que más allá de saber qué y cómo proceder, lo relevante es integrar la teoría y práctica para 
resolver las dificultades experimentadas. Por el contrario, la falta de comunicación en la tríada contribuye a la disociación universidad-escuela. En consecuencia, no hay convergencia entre criterios para la evaluación del proceso de tutoría (Tejada-Fernández, Carvalho-Dias y RuizBueno, 2017).

De este modo, el estudiantado de IP reconoce que la universidad debe generar canales de comunicación hacia ellas y ellos. Estos espacios de comunicación son necesarios desde el inicio de la práctica profesional, como un mecanismo de apoyo frente a las dificultades que experimenta la población estudiantil de IP:

...por parte de la universidad, apoyar a los estudiantes, ver diversos conflictos que pueden darse dentro del aula. Porque no hay un ramo asociado a eso, a nosotros nos entregan la teoría, todo hermoso, pero la realidad es otra y los contextos son diferentes. Quizás tener una asignatura que enseñe a los practicantes a cómo enfrentar diversos conflictos, situándose en diferentes contextos... (Entrevista 04:20).

En atención a lo anterior, la universidad debe formalizar el contexto escolar como un espacio formativo. Es decir, considerar el contexto escolar como un campo de co-formación y aprendizaje para todas y todos (Gervais y Desrosiers, 2005). Por el contrario, la experiencia de acompañamiento de estudiantes de IP desvela la insuficiente capacidad de la institución formadora para recoger aquellos aprendizajes prácticos que se originan en las instituciones escolares. Esto contribuiría a sistematizar, formalizar y validar el contexto escolar como escenario de aprendizajes situados.

\subsubsection{Relación tutor o tutora-estudiante}

En esta subcategoría se describen problemáticas que surgen en la relación entre profesor o profesora, tutores, tutoras y estudiante de IP. Se trata de problemáticas que influyen en la práctica profesional. La primera de ellas es la falta de apoyo y ausencia del docente que desarrolla la tutoría en la sala de clases. Además, otra dificultad en la relación docente-tutor o tutora y estudiante de IP es la experimentación de abusos, y falta de espacios de reflexión sobre las prácticas pedagógicas realizadas por el estudiantado.

La falta de apoyo del docente tutor o tutora se expresa en la desatención sobre los diseños de enseñanza propuestos por estudiantes de IP. En consecuencia, se sienten desorientados y confundidos “....ahí [diseño de planificación] me dejó sola (...) me sentía en situaciones desorientada, porque no tenía tampoco planificaciones, al final empecé a planificar 
siguiendo el programa de estudio..." (Entrevista 05:06). Por lo cual, el acompañamiento se torna una contradicción en sí mismo al no propiciar una coordinación, en este caso pedagógica, entre docentes y estudiantes (Bretones, 2013). Esto convierte al acompañamiento en un conjunto de improvisaciones para estudiantes de IP.

La falta de apoyo implica la ausencia del profesorado tutor en sala de clases. Así, estudiantes de IP se desempeñan en una situación de orfandad. Sumado a esto se enfrentan a una multiplicidad de tareas y responsabilidades que no necesariamente forman parte de sus competencias:

...muchas veces me tocó estar sola en la sala. Porque había muchas problemáticas del colegio, entonces tenía [profesora tutora] que salir mucho de la sala de clases, y bueno para mí me sirvió, porque me dio más experiencia. Pero había muchas situaciones en las que me sentí sola, por ejemplo, un día se me accidentó un niño. (Entrevista 05:02).

Para los centros de práctica, la población estudiantil de IP representa un recurso para cubrir necesidades personales del profesorado y/o de la institución escolar. Según Jiménez (2017), en estas situaciones el estudiantado en práctica se convierte en suplentes del profesor o profesora titular. Esto concuerda con el modelo de acompañamiento nada o húndete (Vonk, 1994) en el cual se genera una situación de soledad que priva a estudiantes de la orientación del docente que desarrolla la tutoría.

Junto a lo anterior, la falta de instancias para la reflexión del quehacer pedagógico del estudiante de IP genera dificultades para mejorar las intervenciones pedagógicas. Esto contribuye a sentimientos de inseguridad en estudiantes, puesto que no hay sugerencias pedagógicas que fortalezcan su desempeño. De acuerdo con los estudiantes de IP esta problemática se debe a un exceso de libertad: “...creo que no había retroalimentación una vez que hacía las clases (...) porque creo me dio exceso de libertad, si en realidad en las primeras dos clases creo que me dijo alguna sugerencia, después no" (Entrevista 03:03). Esto contrasta con las condiciones de acompañamientos de excelencia. En efecto, la calidad de las prácticas pedagógicas iniciales supone acompañar la reflexión teórica sobre las prácticas (Blanco y Latorre, 2011; Schön, 2002). Por lo tanto, la ausencia de retroalimentación genera prácticas de acompañamiento irreflexivas.

En la relación entre tutores o tutoras y estudiantes de IP surgen conflictos en el contexto de prácticas cotidianas en la escuela. Por ejemplo, al estudiantado en práctica se les sugiere no compartir espacios junto al profesorado. También experimentan sobrecarga de tareas 
administrativas de responsabilidad de personas tutoras. Estas situaciones comprenden un modo violento de relación que ejerce el profesorado tutor hacia estudiantes de IP:

Era como su hijo, por eso tenía que pedirle disculpas por todo (...) me retaba súper fuerte como si fuera una mamá, me decía ¡¿por qué hiciste esto, pero por qué?!...disculpe, no se va a volver a repetir le decía yo, pero por favor discúlpeme, pero de verdad profesora, le decía para que se calme. Súper sumiso todos los días... siempre me decía, ya yo te disculpo, y ahí yo la abrazaba. (Entrevista 02:10).

De acuerdo con Torrego (2008), un estilo formalista y autoritario puede resultar en una confrontación entre profesorado-tutor y estudiante. De esta manera, la reacción frecuente en estudiantes es anular psicológicamente a tutores y tutoras De este modo, estudiantes de IP construyen la imagen de una relación dada solo por compartir un espacio físico. Lattanzi y Vanegas (2020) reconocen la importancia de la práctica profesional para la interacción del estudiante con otros docentes. Esto fortalece el conocimiento de la cultura escolar.

El estudiantado de IP atribuye que el modo de relación con profesorado-tutor responde al miedo o amenaza que representa la figura de estudiante para la continuidad laboral de docentes. En efecto, al finalizar el año escolar estudiantes de IP pueden ser contratados por la institución escolar, y de esta manera ocupar el lugar del docente que desarrolló la tutoría:

Pero cuál es el incentivo para que el profesor tutor lo deje bien formado (al estudiante en práctica) ¿para que al año siguiente me quite la pega? (...) al finalizar el segundo semestre la relación fue muy crítica (...) en el sentido de decirme ¡envíame la prueba!, ¡no me has enviado esto!, ¡envíame la guía!... porque ya se acercaba el tiempo de los contratos. (Entrevista 01:30)

Lo descrito contraviene todo el sentido e intencionalidad del acompañamiento pedagógico. En primer lugar, pone en peligro la relación intersubjetiva entre docentes y estudiantes, y los procesos de transferencia e implicación mutua (Ardoino, 2005). En segundo lugar, se transgrede el principio de mediación para la construcción de aprendizajes y desarrollo de actitudes y conocimiento científico en estudiantes (Lobato, 2004). En definitiva, el rechazo mutuo altera toda ética de la formación, con lo cual no es posible el acompañamiento. 


\subsubsection{Características socioculturales del alumnado}

Otro aspecto que tensiona el desempeño de estudiantes de IP son las características socioculturales del alumnado. Estas características responden a particularidades del entorno social y familiar en el que se encuentran a diario. Por ejemplo, situaciones de abandono, maltrato, adicciones, entre otras, forman parte de la experiencia de alumnos y alumnas que luego afectan las relaciones con sus profesores y profesoras:

...el contexto de vulnerabilidad se expresaba en la conducta de los estudiantes, en como llegaban al colegio, en las características que tenía cada uno en su entorno (...) Tenían muchos problemas, problemas con los papás [padres], problemas de abusos, de violencia física, sexual, anorexia, bulimia. (Entrevista 04:01).

El estudiantado reconoce que las situaciones vividas por alumnos y alumnas se traducen en desmotivación frente a la enseñanza. Esto da origen a expectativas laborales que se distancian:

Lo que más pude ver fue el desinterés de los estudiantes, la desmotivación (...) en primero medio [primer año secundaria] había una gran problemática, porque la gran mayoría, más del cincuenta por ciento estaba quedando repitiendo, y algunos se retiraron en el proceso, y algunos se iban a trabajar al campo, y era como eso, no estaban muy motivados. (Entrevista 03:01)

Las prácticas de acompañamiento descritas muestran un choque con la realidad (Jiménez, 2017) que supone la transgresión de las expectativas del futuro profesorado. Efectivamente, la vocación se pone en cuestionamiento cada vez que el estudiante de IP no encuentra las herramientas adecuadas para resolver problemáticas en la sala de clases. Nuevamente, esto es producto de un acompañamiento insuficiente, donde el tutor o tutora es un actor marginal en el desempeño del estudiante de IP.

\subsection{Criterios de mejoramiento para las prácticas de acompañamiento}

En esta categoría se evidencian criterios que permiten mejorar prácticas de acompañamiento realizadas por profesorado tutor. De esta manera, se plantean criterios de selección para los encargados de las tutorías e incentivos que mejoren su compromiso hacia el acompañamiento. 


\subsubsection{Selección de tutores}

La selección de tutores o tutoras es una condición que estudiantes de IP consideran necesaria para garantizar su idoneidad. En este sentido, la universidad debe asegurar docentes que ejerzan la tutoría cumplan con requisitos mínimos para acompañar al estudiante de IP. En consecuencia, se debe crear y socializar un perfil que oriente la selección y el rol de la persona tutora durante el proceso de acompañamiento:

...ellos [universidad] se encargan de buscar los colegios, pero creo que podrían ir más allá, qué profesores van a recibir a los estudiantes, porque se fijan en los establecimientos, pero no saben que profesores están ejerciendo, yo creo que podrían realizar charlas, un taller a los profesores antes de recibir a los estudiantes, decirles; esto es lo que queremos que hagan con los estudiantes, para que ellos sepan también, porque no saben qué hacer... (Entrevista 05:21).

De acuerdo con estudiantes de IP es conveniente establecer criterios de selección para un perfil docente de personas tutoras. En efecto, las observaciones de procesos de acompañamiento realizados por González et al (2017) determinan que todavía falta elaborar un perfil idóneo para la tutoría. Esto permitiría a la universidad realizar una evaluación del profesorado que está en condiciones de llevar a cabo el acompañamiento. Esta problemática se confirma en un estudio reciente que constata la definición ambigua del rol de la tutoría, lo cual relega la responsabilidad de la acción tutorial en manos del tutor o tutora (Latorre et al., 2020).

Sin duda la selección de tutores y tutoras permite fijar claramente sus funciones, pero además emplear evaluaciones de estudiantes en práctica que luego contribuyan a esta selección (González y Hevia, 2011).

Junto a esto, se evidencia la importancia del compromiso asumido por docentes-tutores o tutoras. Es decir, se considera fundamental que el cuerpo de docente tenga la disposición para llevar a cabo el acompañamiento. Por el contrario, participar en el acompañamiento de estudiantes de IP es considerado por el profesorado que realiza la tutoría como un mandato institucional:

...realizarle una evaluación al profesor tutor, ver si está capacitado o no... una evaluación previa para ver si cumple con los criterios... y ver si él quiere o no, porque en el caso de la profesora que nos tocó no se sentía cómoda, y tal vez la estaban obligando a tenernos... (Entrevista 02:23). 
Las tensiones que originan la demanda por una profesionalización del acompañamiento surgen como un síntoma de la falta de políticas y programas para la formación de tutores y tutoras (Cornejo, 2014). Sobre todo, esta demanda de estudiantes en práctica da cuenta de la inercia que ha mostrado la universidad frente a las experiencias de acompañamiento de los futuros maestras y maestros.

\subsubsection{Incentivo tutoría}

El estudiantado de IP propone que el profesorado-tutor debe ser incentivado. En este caso, se plantean incentivos financieros que, de alguna manera, retribuyan el tiempo dedicado al acompañamiento. En efecto, se trata de tiempo dedicado a la formación del futuro profesorado, por tanto, esto debe incluir mecanismos de reconocimiento al compromiso. De acuerdo con Adaros (2014), la eficacia del acompañamiento se relaciona con la responsabilidad con la cual quienes ofrecen la tutoría asumen el compromiso formativo hacia el futuro de la persona docente.

Otras formas de incentivos se relacionan con reconocimiento profesional. Por ejemplo, la difusión de experiencias de acompañamiento en contextos escolares y/o académicos representa un reconocimiento a la labor de la tutoría: “...que se les brinde un incentivo económico, que la universidad al menos les invite (...) a una capacitación, (...), que se realice un conversatorio y que participen con una ponencia (...) sobre su experiencia..." (Entrevista 01:32). En este sentido, Méndez (2012) sostiene que la coordinación entre universidad y centros de prácticas tienen que formar escuelas de desarrollo profesional. Estas escuelas se deben nutrir de la investigación reflexiva y perfeccionamiento continuo entre supervisores, profesoras y profesores, tutores y tutoras y estudiantes de IP.

Lo anterior coincide con los hallazgos de Ruffinelli, et al (2020), que sugieren profesionalizar la tutoría e incorporar a nuevos actores en ella para lograr una co-construcción reflexiva y colaborativa en los centros educativos. En el mismo sentido, Latorre, et al. (2020) reconocen que la formación y especialización de tutores y tutoras son desafíos importantes para mejorar las prácticas de acompañamiento.

\section{Conclusiones}

Las siguientes conclusiones responden a la realidad descrita para el caso de la institución donde se realizó el estudio. Entonces, de acuerdo con los resultados analizados, es necesario repensar el rol del docente tutor o tutora e inscribirlo en un marco de 
acompañamiento profesional. De este modo, será posible resolver la situación de abandono de estudiantes en práctica pedagógica. Consideramos que esta problemática tiene su origen en la propia institución formadora, y se expresa en la relación profesorado, tutor o tutora y estudiante de IP.

La experiencia de estudiantes de IP se encuentra en una encrucijada que les obliga a responder a exigencias disímiles. Por una parte, la institución formadora se empeña en que el futuro cuerpo docente cumpla con estándares que se muestran asincrónicos frente al trabajo pedagógico requerido por las escuelas. Por ejemplo, las actividades exigidas por la universidad son percibidas como burocráticas para un escenario escolar que requiere de dinamismo para resolver problemas prácticos. Por otra parte, la relación entre institución formadora y centro de práctica requiere de coordinación efectiva. De acuerdo con los resultados, tanto el profesorado-tutor como el estudiantado de IP desconocen sus roles. Junto a esto, la institución formadora ignora la cultura escolar.

Esta falta de claridad respecto de cómo llevar a cabo un acompañamiento profesional nos sitúa en prácticas de acompañamiento artesanales. Es decir, la improvisación, falta de rigor, ausencia de objetivos claros, imposibilidad de diálogo universidad - centros educativos y estudiantes de IP, nos hacen pensar que se requiere revisar, si es que existen, políticas de acompañamiento impulsadas por las universidades.

Se considera que el acompañamiento pedagógico es clave, no solo como instancia que consolida la formación realizada en la educación superior, sino que también es crucial para la construcción de identidad docente. Por tanto, un acompañamiento pedagógico que responda a las complejidades que enfrentan estudiantes en prácticas pedagógicas fortalece la seguridad en la toma de decisiones y, por tanto, la autoestima del futuro profesorado. Por contrario, la deficiencia en el acompañamiento pedagógico produce sentimientos de angustia e inseguridad que pueden traducirse en frustración profesional hasta socavar la vocación docente.

El acompañamiento se traduce en un conjunto de acciones que responden a la necesidad de cumplir exigencias que no convergen entre sí. Sumado a esto, la selección de tutores y tutoras debe estar acompañada de criterios claros e incentivos que, al menos, permitan contar con el compromiso de formar a futuros docentes.

Desde la investigación educativa será necesario formar redes de colaboración no solo a nivel regional y nacional, sino también a nivel internacional, de manera de abrir espacios de análisis, diálogo y reflexión sobre experiencias formativas de estudiantes en práctica pedagógica. En nuestra calidad de equipo de investigación esperamos consolidar instancias 
de intercambio de experiencias y contribución investigativa sobre el tema de acompañamiento pedagógico. En efecto, nos ponemos a disposición de la comunidad educativa interesada en avanzar y contribuir a la comprensión del tema descrito en este estudio.

Este estudio presenta limitaciones de orden metodológico en atención a la imposibilidad de acceder a la experiencia de participantes relacionados con tutorías y supervisiones. Esto permitirá fortalecer los criterios que se presentan en los resultados de este estudio, y en términos metodológicos, facilitará la triangulación de perspectivas.

Finalmente, se recomienda elaborar un perfil de tutor o tutora donde participen universidad, centros educativos y estudiantes de IP. De lo contrario, seguiremos reproduciendo un acompañamiento que contribuye a profundizar la brecha entre teoría y práctica pedagógica.

\section{Agradecimiento}

El equipo de investigación que elaboró este artículo, agradece el financiamiento recibido por parte de la Vicerrectoría de Investigación y Posgrado de la Universidad Católica de Temuco, Chile.

\section{Referencias}

Adaros, Marcela. (2014). Acompañamiento pedagógico recibido por alumnos de pedagogía a través de las bitácoras en el contexto de la práctica profesional. Revista de Estudios y Experiencias en Educación, 13(26), 91-116. Recuperado de http://www.rexe.cl/ojournal/index.php/rexe/article/view/36/38

Alliaud, Andrea. (2017). Los artesanos de la enseñanza. Acerca de la formación de maestros con oficio. Argentina: Paidós.

Arbizu, Feli., Lobato, Clemente., y Castillo, Laura. (2005). Algunos modelos de abordaje de la tutoría universitaria. Revista de Psicodidáctica, 10(1), 7-22. Recuperado de: http://www.researchgate.net/publication/39217715 Algunos modelos de abordaje de la tutoria universitaria

Ardoino, Jacques. (2005). Complejidad y formación. Pensar la educación desde una mirada Epistemológica. Buenos Aires: Facultad de Filosofía y Letras. UBA Ed. Novedades Educativas.

Arguis, Ricardo., Pere, Arnaiz., Báez, Coral., De Ben, Miguel., Díaz, Fernando., Diez, María... Vicente, Auxilio. (2011). La acción tutorial. El alumnado toma la palabra. Venezuela: Laboratorio Educativo.

Ariza, Gladys., y Ocampo, Héctor. (2005). El acompañamiento tutorial como estrategia de la formación personal y profesional: un estudio basado en la experiencia de una institución de educación superior. Universitas Psychologica, 4(1), 31-41. Recuperado de: http://www.redalyc.org/articulo.oa?id=64740104 
Ávalos, Beatrice. (2004). La formación docente inicial en Chile. Santiago. Recuperado de: http://www.ub.edu/obipd/PDF\%20docs/Formaci\%C3\%B3\%20Inicial/Educaci\%C3\%B3\% 20Universitaria/Documents/La\%20Formacion\%20Docente\%20Inicial\%20en\%20Chile. \%20AVALOS.pdf

Banister, Peter., Burman, Erika., Parker, lan., Taylor, Maye. y Tindall, Carol. (2004). Métodos Cualitativos en Psicología. México: Universidad de Guadalajara.

Benoit, Claudine. (2021). La tutoría entre pares: una estrategia para el fortalecimiento de la vocación pedagógica. Transformación, 17(1), 1-17. Recuperado de http://scielo.sld.cu/scielo.php?script=sci arttext\&pid=S2077$29552021000100001 \& \operatorname{lng}=$ es\&tlng=es

Blanco, Francisco., y Latorre, María. (2011). El prácticum como espacio de aprendizaje profesional para docentes en formación. Revista de docencia universitaria, 9(2), 35-54. Recuperado de http://red-u.net/redu/files/journals/1/articles/269/public/269-532-3-PB.pdf

Bretones, Antonio. (2013). El Prácticum de magisterio en educación primaria: una mirada retrospectiva. Revista Complutense de Educación, 24(2), 443-471. Recuperado de: http://dx.doi.org/10.5209/rev RCED.2013.v24.n2.42088

Bullough, Robert. (2000). Convertirse en profesor: la persona y la localización social de la formación del profesorado. En Biddle, Good y Goodson (eds.), La enseñanza y los profesores I. La profesión de enseñar (pp. 99-165). Barcelona: Paidós.

Canales, Manuel. (2013). El diseño en estudios ideográficos. En Manuel Canales (Ed.), Metodologías de investigación social introducción a los oficios. (pp. 193-206). Santiago: LOM.

Canales, Manuel. (2017). Metodologías de investigación social. Introducción a los oficios. Chile: LOM.

Cano, Rufino. (2008). Modelo organizativo para la planificación y desarrollo de la tutoría universitaria en el marco del proceso de convergencia europea en Educación Superior. Revista Interuniversitaria de Formación del Profesorado, 22(61), 185-204. http://www.researchgate.net/publication/43164125 Modelo organizativo para la planif icacion y desarrollo de la tutoria universitaria en el marco del proceso de conve rgencia europea en Edcacion Superior

Carnoy, Martín. (2010). La ventaja académica de Cuba. Por qué los estudiantes cubanos rinden más. México: Fondo de Cultura Económica.

Cid, Alfonso., Pérez, Adolfo., Sarmiento, José. (2011). La tutoría en el prácticum. Aportaciones de los simposios de Poio. Santiago de Compostela: Andavira.

Cornejo, José. (2014). Prácticas profesionales durante la formación inicial docente: análisis y optimización de sus aportes a los que aprenden y a los que enseñan a aprender a enseñar. Estudios Pedagógicos, 40(Especial), 239-256. Recuperado de: http://scielo.conicyt.cl/scielo.php?script=sci arttext\&pid=S0718-07052014000200014 
Cox, Cristian y Gysling, Jaqueline. (1990). La formación del profesorado en Chile 1842-1987. Chile: Centro de Investigación y Desarrollo de la Educación.

Denzin, Norman., y Lincoln, Yvonna. (2015). Métodos de recolección y análisis de datos. Argentina: Gedisa.

Dewey, John. (1988) La escuela y el progreso social. Recuperado de: http://www.unav.es/gep/Dewey/EscuelaProgresoSocialBILE.html

Donnay, Jean., y Charlier, Evelyn. (2006). Apprendre par l'analyse des pratiques. Initiation au compagnonnage réflexif. Belgique: Editions du CPP, Presses Universitaire de Namur.

Eirín-Nemiña, Raul. (2018). Las comunidades de aprendizaje como estrategia de desarrollo profesional de docentes de Educación física. Estudios pedagógicos, 44(1), 259-278. Recuperado de https://scielo.conicyt.cl/pdf/estped/v44n1/0718-0705-estped-44-0100259.pdf

Elías, María. (2015). La cultura escolar: Aproximación a un concepto complejo. Educare, 19(2), 285-301. Doi http://dx.doi.org/10.15359/ree.19-2.16

Estay, Christians. (2011). Investigación cualitativa en sistemas de información. Recuperado de http://cestay.wordpress.com/tag/investigacion-cualitativa-en-sistemas-de-informacion/

Flick, Uwe. (2019). Introducción a la investigación cualitativa. España: Morata.

García Nieto, Narciso. (2008). La función tutorial de la universidad en el actual contexto de la educación superior. Revista interuniversitaria de formación del profesorado, 22(1), 21 48. Recuperado de http://dialnet.unirioja.es/descarga/articulo/2541038.pdf

Gervais, Colette., y Desrosiers, Pauline. (2005). L'école, lieu de formation d'enseignants. Questions et repères pour l'accompagnement de stagiaires. Canadá: Les Presses de l'Université de Laval.

Gibbs, Graham. (2009). Analyzing Qualitative Data. London: Sage.

Gimeno, Miguel. (2014). Cierre de las escuelas normales en Chile. Cuadernos de historia de Chile, (2), 119-130. Recuperado de http://www.academia.edu/26198668/Miguel Gimeno Cierre de las Escuelas Normal es en Chile 1973 - 1974 . Decretos N 179 y 353

González, Xosé., y Hevia, Isabel. (2011). El practicum de la licenciatura de pedagogía: Estudio empírico desde la perspectiva del alumnado. Revista de Educación, (354), 209-236. Recuperado de http://www.educacionyfp.gob.es/revista-de-educacion/en/numerosrevista-educacion/numeros-anteriores/2011/re354/re354-09.html

González, Nora., Uvalle, Yolanda., y Hernández, María. (2017). El acompañamiento pedagógico como función tutorial para los futuros docentes: Hacia un enfoque socioformativo. Congreso nacional de investigación educativa. Recuperado de http://comie.org.mx/congreso/memoriaelectronica/v14/doc/0920.pdf 
Harvard Business School. (2004). Coaching y Mentoring. Bilbao: Ed. Deusto.

Herrera, José., y Martínez, Ángela. (2018). El saber pedagógico como saber práctico. Pedagogía y Saberes, 49, 9-26. Recuperado de http://www.scielo.org.co/pdf/ppo/n19/2011-804X-ppo-19-00009.pdf

Hevia, Isabel y Fueyo, Aquilina. (2018). Aprendizaje situado en el diseño de entornos virtuales de aprendizaje una experiencia de aprendizaje entre pares en una comunidad de práctica. Aula abierta, 47(3), 347-354. Recuperado de https://dialnet.unirioja.es/servlet/articulo?codigo $=6723286$

Hock, Michael y Pulvers, Kim. (2001). The effects of an after school tutoring program on the academic performance of at risk student and students with L.D. Remedial and Special Education. PRO-ED. Journals, 22(3), 172-186. Recuperado de: https://pdfs.semanticscholar.org/a0b9/45bd2a41ee6d81a0528c8e8d29565d20e907.pdf

Jiménez, María de la Luz. (2017). Experiencias de acompañamiento en la formación docente. Supuestos, imaginarios y prácticas. En Conisen, Memorias del congreso nacional de educación normal (pp. 1-13; México). Recuperado de http://www.conisen.mx/memorias/memorias/5/C180117-H113.docx.pdf

Lattanzi, Romina., y Vanegas, Carlos. (2020). Acompañamiento de prácticas pedagógicas de formación inicial: un self-study a partir del rol como profesora guía. Revista Iberoamericana De Educación, 82(1), 141-160. Recuperado de https://doi.org/10.35362/rie8213660

Latorre, Marisol., Vergara, Claudia., Morales, María., Orbeta, Alejandra., Escobar, Claudia., y Quiroga, Marta. (2020). Prácticas de acompañamiento de profesores tutores en carreras de pedagogía: análisis de los dispositivos de aseguramiento y certificación de la calidad de los aprendizajes terminales. Calidad en la Educación, (53), 182-218. doi: https://doi.org/10.31619/caledu.n53.791

Lobato, Clemente. (2004). La función tutorial universitaria: estrategias de intervención. Papeles Salmantinos de Educación, 3, 32-57. Recuperado de http://www.researchgate.net/publication/264874200 LA FUNCION TUTORIAL UNIVE RSITARIA ESTRATEGIAS DE INTERVENCION

Lunenberg, Mieke., Dengerink Jurriën., and Korthagen, Fred. (2014). The Professional Teacher Educator. Roles, Behaviour, and Professional Development of Teacher Educators. Rotterdam: Sense Publishers.

Mansilla, Juan. (2018). Influencia alemana en la reforma de las Escuelas Normales de Preceptores y Preceptoras en el centro sur de Chile, 1883-1920. Revista Historia de la Educación Latinoamericana, 31(20), 189-209. Doi https://doi.org/10.19053/01227238.8574

Marcelo, Carlos. (2008). Profesores principiantes e inserción a la docencia. Barcelona: Ediciones Octaedro. 
Méndez, Laura. (2012). El conocimiento situado y los sistemas de actividad. Un modelo teórico para repensar el practicum. Revista de Educación, (359), 629-642. Recuperado de http://dialnet.unirioja.es/servlet/articulo?codigo $=4015551$

Moore, Will. (1968). The Tutorial System and its Future. Oxford: Pergamond Press.

Munchi, Anicha. (2018). Induction Programs, Teacher Efficacy, and Inquiry Practices in Novice Teachers. EEUU: San Jose State University.

Núñez, Ivan. (2002). La formación de docentes. Notas históricas. En Ávalos, Beatrice, Profesores para Chile. Historia de un proyecto (pp. 15-39). Santiago: Ministerio de Educación.

Páez, Ruth. (2015). Práctica y experiencia: claves del saber pedagógico docente. Ediciones Unisalle: Bogotá.

Perrenoud, Philippe. (2004). Diez nuevas competencias para enseñar. Barcelona: Grao.

Ruffinelli, Andrea., Morales, Alejandra., Montoya, Soledad., Fuenzalida, Claudia., Rodríguez, Claudia., López, Patricia. y González, Carolina. (2020). Tutorías de prácticas: representaciones acerca del rol del tutor y las estrategias pedagógicas. Perspectiva educacional. 59(1), 30-51. Recuperado de http://www.perspectivaeducacional.cl/index.php/peducacional/article/viewFile/1004/336

Ruiz, José. (2012). Metodología de investigación cualitativa. España: Universidad de Deusto.

Sánchez, Juan. (2016). El carácter bipolar del prácticum de magisterio: un reto para la innovación. Opción. Revista de Ciencias Humanas y Sociales, 32(10), 715-733. Recuperado de: http://dialnet.unirioja.es/servlet/articulo?codigo=5875190

Sánchez- Amaya, Tomás y González- Melo, Hamlet. (2016). Saber pedagógico: fundamento del ejercicio docente. Educación y educadores. 19(2), 241-253. Recuperado de https://www.redalyc.org/pdf/834/83446681004.pdf

San Martín, Daniel. (2014). Teoría fundamentada y Atlas.ti: recursos metodológicos para la investigación educativa. Revista Electrónica de Investigación Educativa, 16(1), 103-122. Recuperado de http://redie.uabc.mx/vol16no1/contenido-sanmartin.html

Schön, Donald. (2002). La formación de profesionales reflexivos. Hacia un nuevo diseño de la enseñanza y el aprendizaje en las profesiones. España: Editorial Paidós.

Slavin, Robert. (1991). Student Team Learning: A Practical Guide to Cooperative Learning. Washington DC: National Education Association.

Steiner, George. (2011). Lecciones de los maestros. España: Ediciones Siruela.

Strauss, Anselm., y Corbin, Juliet. (2002). Bases de la investigación cualitativa. Técnicas y procedimientos para desarrollar la Teoría Fundamentada. Colombia: Universidad de Antioquia. 
Maxwell, Joseph. (2019). Diseño de investigación cualitativa. Barcelona: Gedisa.

Tejada, José. (2006). Elementos de convergencia entre la formación profesional y la universidad: Implicaciones para la calidad de la formación profesional superior. Revista de educación, (340), 1085-1117. Recuperado de: https://www.researchgate.net/publication/28158235 Elementos de convergencia entr e la formacion profesional y la universidad implicaciones para la calidad de la $f$ ormacion profesional superior

Tejada-Fernández, José., Carvalho-Dias, María., y Ruiz-Bueno, Carmen. (2017). El prácticum en la formación de maestros: percepciones de los protagonistas. Magis Revista Internacional de Investigación en Educación, 9(19), 91-114. doi: https://doi.org/10.11144/Javeriana.m9-19.pfmp

Torrego, Juan Carlos. (2008). El profesor como gestor en el aula. En Agustín Herrán y Joaquín Paredes (ed.), Didáctica general: la práctica de la enseñanza en Educación Infantil, Primaria y Secundaria (pp. 197-213). España: McGraw-Hill.

Trinidad, Antonio., Carrero, Virginia., y Soriano, Rosa María. (2006). La construcción de la teoría a través del análisis interpretacional. España: Centro de Investigaciones Sociológicas.

Valles, Miguel. (1997). Técnicas cualitativas de investigación social. Reflexión metodológica y práctica social. España: Síntesis.

Vonk, Hans. (1994). Teacher Induction: The great omission in education. En Maurice Galton y Bob Moon (eds.), Handbook of Teacher Training in Europe (pp. 85-108). Londres: David Fulton.

Woolf, Nicholas., y Silver, Chirstina. (2018). Qualitative Analysis Using ATLAS.ti: The FiveLevel QDATM Method. New York: Routledge. 
Revista indizada en

sciplo

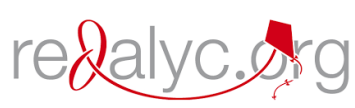

latindex

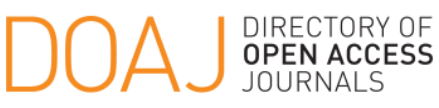

Distribuida en las bases de datos:

○ Dialnet

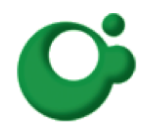
SHERPA/RoMEO

REDIB

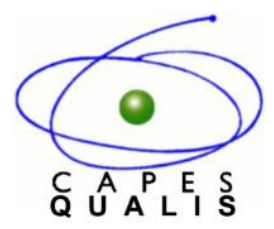

MIAR 